\title{
Combined sticking: a new approach for finite-amplitude Coulomb frictional contact
}

\author{
P. Areias $(\mathbb{D} \cdot$ F. Queirós de Melo · J. Carrilho Lopes
}

Received: 24 August 2019/ Accepted: 22 October 2019

(C) Springer Nature B.V. 2019

\begin{abstract}
Engineering-level accuracy of discretization methods for frictional contact originates from precise representation of discontinuous frictional and normal interaction laws and precise discrete contact techniques. In terms of discontinuous behavior in the quasi-static case, two themes are of concern: the normal interaction (i.e. impact) and the jumps in tangential directions arising from high frictional values. In terms of normal behavior, we use a smoothed complementarity relation. For the tangential behavior, we propose a simple and effective algorithm, which is based a stick predictor followed by corrections to the tangential velocity. This allows problems with impact and stick-slip behavior to be
\end{abstract}

\section{P. Areias}

DEM - Departamento de Engenharia Mecânica, Instituto Superior Técnico, Universidade de Lisboa, Avenida Rovisco Pais, 1049-001 Lisbon, Portugal

P. Areias $(\square)$

IDMEC - Instituto Superior Técnico, Universidade de Lisboa, Lisbon, Portugal

e-mail: pedro.areias@tecnico.ulisboa.pt

F. Q. de Melo

INEGI - Instituto de Ciência e Inovação em Engenharia Mecânica e Engenharia Industrial, Rua Dr. Roberto Frias 400, 4200-465 Porto, Portugal

J. C. Lopes

DGEO - Departmento de Geociências, Colégio Luís António Verney, Universidade de Évora, Rua Romão Ramalho, 59, 7002-554 Évora, Portugal solved with an implicit code based on NewtonRaphson iterations. Three worked examples are shown with comparisons with published results. An extension to node-to-face form in 3D is also presented.

Keywords Frictional contact $\cdot$ Finite strain $\cdot$ Stick predictor $\cdot$ Smoothing

\section{Introduction}

The precise representation of mechanical contact between surfaces, modeled via unilateral constraints, is still one of the most challenging tasks in computational mechanics. Several difficulties are present within the context of implicit solvers:

1. Non-smoothness of contact forces due to the conecomplementarity conditions, discussed by Kanno et al. (2006). Artificial smoothing of the conditions will result in absence of stick-slip oscillations (which are observed experimentally) even for high values of the coefficient of friction.

2. Non-smoothness of contact forces due to the facetlike geometry characteristic of finite element discretizations, addressed by Puso and Laursen (2004b).

3. When ad-hoc algorithms are used, energy errors and spurious noise appear (Khenous et al. 2008). 
We are here concerned with item 1.) and propose simple solutions for these difficulties. Historically, experimental observations by Amontons (1699) produced the dry frictional laws. This theory was concluded by Coulomb (1821) who postulated the independence of kinetic friction force with respect to the relative velocity. This preliminary theory was essentially discrete (for discrete case, the review by Stewart proves the presence of paradoxes Stewart 2001) and the continuum case was completed much later by Kikuchi and Oden (1988). Contributions to discrete friction were performed by Martins et al. (1995), De Saxcé and Feng (1998) and Wriggers and Haraldsson (2003). We are here concerned with smoothing algorithms that converge to the original non-smooth conditions and with the development of stable algorithms to ensure that the jumping phenomena in time are properly represented. From a computational perspective, variations of the augmented Lagrangian method have been explored, with firstorder updating (see Simo and Laursen 1992) and second order updating (see Alart and Curnier 1992). The dealing of friction with the augmented Lagrangian method involves the operator split technique (cf. Wriggers 2002), see also Laursen 2002; Wriggers 2002. Refaat and Meguid developed an effective algorithm based on the sequential solution of two special cases (Refaat and Meguid 1995), which was found to be convergent for Hertz-type contact with friction. Another aspect, not addressed here, is the one of discretization (see Wriggers 2002 for a comprehensive treatment), which has recent developments, cf. (Anitescu et al. 2019).

As an alternative, smoothing of the complementarity conditions in frictional contact was performed by Areias et al. (2014). A related topic is the one of friction models which can be viewed as regularization (Pennestri et al. 2016).

We here do not regularize the frictional behavior, but use a stick predictor followed by a slip behavior that progressively approaches (and reaches) the original Coulomb law. In terms of discretization, which we briefly describe the $3 \mathrm{D}$ case where the node-to-face approach is used. We use Acegen (Korelc 2002), in combination with Mathematica software (Wolfram Research Inc. 2007) to derive the closed-form expressions.

\section{Governing equations for a plane, rigid obstacle}

The Signorini problem combined with classical Coulomb friction is first considered with the plane $x_{3}=0, \boldsymbol{e}_{3}=\{0,0,1\}^{T}$ being the obstacle. This assumption simplifies this description without limiting further generalization, performed in the subsequent section. We introduce the normal force as the product of a scalar multiplier $\gamma_{n}$ with the normal direction $\boldsymbol{e}_{3}: \boldsymbol{f}_{n}=\gamma_{n} \boldsymbol{e}_{3}$. Using a tangent subspace with basis vectors $\boldsymbol{e}_{1}, \boldsymbol{e}_{2}, \quad$ (here $\boldsymbol{e}_{1}=\{1,0,0\}^{T}$ and $\boldsymbol{e}_{2}=$ $\{0,1,0\}^{T}$ forming the canonical basis) then the tangential force can be written as $\boldsymbol{f}_{t}=\gamma_{1} \boldsymbol{e}_{1}+\gamma_{2} \boldsymbol{e}_{2}$ with $\gamma_{1}$ and $\gamma_{2}$ being the two force components. The total contact force is a sum of both contributions: $\boldsymbol{f}_{c}=\boldsymbol{f}_{n}+\boldsymbol{f}_{t}$. A unique coefficient of friction $\mu$ is adopted, for simplicity of treatment. A non-negative variable $\beta$ is introduced, relating the slip velocity $\dot{\boldsymbol{u}}_{t}$ with the friction force $f_{t}$. Two functions are introduced, one function $\Phi$ corresponding to the contact signed distance and another $\Psi$ which can be interpreted as a frictional yield function (here $\Psi>0$ indicates stick and $\Psi=0$ slip):

$\Phi=x_{3}$

$\Psi\left(\gamma_{n}, \boldsymbol{f}_{t}\right)=\mu \gamma_{n}-\left\|\boldsymbol{f}_{t}\right\|$

where $x_{3}=X_{3}+u_{3}$ is the deformed coordinate of the contact point. In this context, $X_{3}$ is the undeformed coordinate of the contact point and $u_{3}$ is the displacement of the same node. Combining the Signorini with Coulomb's frictional conditions we obtain:

$$
\begin{aligned}
& \gamma_{n} \geq 0, \quad \Phi \geq 0, \quad \Phi \gamma_{n}=0 \\
& \beta \geq 0, \quad \Psi \geq 0, \quad \Psi \beta=0 \\
& \dot{\boldsymbol{u}}_{t}+\beta \boldsymbol{f}_{t}=\boldsymbol{0}
\end{aligned}
$$

The first three conditions (3) are the non-penetration complementarity conditions. The second three conditions (4) are the stick/slip friction conditions in terms of the multiplier $\beta$. Finally Eq. (4) relates the tangential velocity with the friction force. This condition can also be written as:

$$
\left\{\gamma_{1}, \gamma_{2}\right\}=\underset{\Psi\left(\gamma_{n}, \tilde{f}_{t}\right) \geq 0}{\operatorname{argmin}}\left[\widetilde{\boldsymbol{f}}_{t} \cdot \dot{\boldsymbol{u}}_{t}\right]
$$


where $\widetilde{\boldsymbol{f}}_{t}$ is the unknown force satisfying $\Psi\left(\gamma_{n}, \tilde{\boldsymbol{f}}_{t}\right) \geq 0$ and minimizing $\tilde{\boldsymbol{f}}_{t} \cdot \dot{\boldsymbol{u}}_{t}$. Effective attempts to solve $(4,5)$ with a classical, non-combinatorial finite element code are inhibited by condition (5). Classically, Eqs. $(3,4)$ can be cast in a different form:

$$
\begin{aligned}
& \left\langle\Phi-\gamma_{n}\right\rangle-\Phi=0 \\
& \langle\Psi-\beta\rangle-\Psi=0
\end{aligned}
$$

where the ramp function is trivially obtained from the absolute value: $\langle\bullet\rangle=\frac{\bullet+|\bullet|}{2}$. At this point, it is trivial produce smoothed replacements of $(7,8)$ but relation (5) cannot be simultaneously satisfied in stick and slip conditions without rewriting of the system. Kanno et al. (2006), eqs. (32-34) presents an analogous result, with a second-order cone linear complementarity problem (SOCLCP) being solved by a smooth solver.

However, a simple analysis reveals the dependence of $\beta$ on the solution:

- Considering $\Psi-\beta<0 \Rightarrow \Psi=0$ implies that slip occurs, and hence $\beta$ could be determined by $\beta=\frac{f_{t} \cdot \dot{u}_{t}}{\dot{\boldsymbol{u}}_{t} \cdot \boldsymbol{u}_{t}}$.

- On the other hand, we have $\Psi-\beta \geq 0 \Rightarrow \beta=0$ implies that $\beta=0 \Rightarrow \dot{\boldsymbol{u}}_{t}=\boldsymbol{0}$.

In both cases, we have a dependence of $\beta$ on $\dot{\boldsymbol{u}}_{t}$ which is predicted since we have three unknown forces and four extra degrees-of-freedom per contact node. Any form of smoothed Newton iteration will be illconditioned for this system. Since there are two distinct behaviors, these are better satisfied in two sequential parts. For a given time-step $I_{t}=\left[T_{i}, T_{i}+\Delta t\right]$, a partition into two sub-steps $I_{t}=$ $I_{\text {stick }} \cup I_{\text {corr }}$ is performed. We have $I_{\text {stick }}=\left[T_{i}, T_{i}+\frac{\Delta t}{2}[\right.$ and $I_{\text {corr }}=\left[T_{i}+\frac{\Delta t}{2}, T_{i}+\Delta t[\right.$. We always start each time step with a stick behavior, then use the stick direction as a "null-velocity" direction. This term is ramped-out to zero so that, at the end of $I_{\text {corr }}$, the correct frictional force is obtained. The residual for stick is then given by:

$$
\boldsymbol{r}_{\text {stick }}=\left\{\begin{array}{c}
-\gamma_{1}^{s} \\
-\gamma_{2}^{s} \\
-\gamma_{n} \\
\dot{u}_{1}^{\star} \\
\dot{u}_{2}^{\star} \\
\left\langle x_{3}-\gamma_{n}\right\rangle-x_{3}=0
\end{array}\right\}
$$

where

$\dot{u}_{k}^{\star}=H\left(\gamma_{n}\right) \dot{u}_{k}$

In (9), $\gamma_{1}^{s}$ and $\gamma_{2}^{s}$ are the stick components of the shear force. Since, for this sub-step, sticking is assumed, these are Lagrange multipliers.

In addition, for slipping, we have:

$$
\boldsymbol{r}_{\text {slip }}=\left\{\begin{array}{c}
-\gamma_{1} \\
-\gamma_{2} \\
-\gamma_{n} \\
\gamma_{1} \sqrt{\dot{w}_{1}^{2}+\dot{w}_{2}^{2}}+\mu \gamma_{n} \dot{w}_{1} \\
\gamma_{2} \sqrt{\dot{w}_{1}^{2}+\dot{w}_{2}^{2}}+\mu \gamma_{n} \dot{w}_{2} \\
\left\langle x_{3}-\gamma_{n}\right\rangle-x_{3}=0
\end{array}\right\}
$$

with

$$
\dot{w}_{k}=(1-\xi) \dot{u}_{k}+\xi \frac{\gamma_{k}^{s}}{\sqrt{\left(\gamma_{1}^{s}\right)^{2}+\left(\gamma_{2}^{s}\right)^{2}}}
$$

We note that, with this formulation (9-12), the only non-smooth equation remaining is the normal complementarity, $\left\langle x_{3}-\gamma_{n}\right\rangle-\Phi=0$ which is replaced by a smooth version:

$$
\left\langle x_{3}-\gamma_{n}\right\rangle_{\varepsilon}-\Phi=0
$$

The function $\langle\bullet\rangle$ is here called smoothed ramp. For this smoothed ramp, we have the Chen-Mangasarian replacement function (Chen and Mangasarian 1995, 1996):

$$
\langle\bullet\rangle_{\varepsilon}= \begin{cases}\bullet+\frac{1}{\alpha} \log [1+\exp (-\alpha \bullet)], & \bullet \geq 0 \\ \bullet+\frac{1}{\alpha}\{-\alpha \bullet+\log [1+\exp (\alpha \bullet)]\}, & \bullet<0\end{cases}
$$

with $\alpha=\frac{\log 2}{\varepsilon}$

The use of this function entails a number of advantages: 
- Removal of inequalities and replacement of nonsmooth equations from the system.

- Control of the convergence behavior by use of parameter $\varepsilon$.

However, two shortcomings are also present:

- A user-defined parameter is now present in the system.

- Very small initial values of $\varepsilon$ can result in loss of convergence in Newton iteration.

We note that with this replacement, contact equations become smooth. Newton iteration is therefore possible for a large enough $\varepsilon$. For consistency of units, we rewrite the system as:

$$
\boldsymbol{r}_{\text {stick }}^{\varepsilon}=\left\{\begin{array}{c}
-\gamma_{1}^{s} \\
-\gamma_{2}^{s} \\
-\gamma_{n} \\
r_{p} \dot{u}_{1}^{\star} \Delta t \\
r_{p} \dot{u}_{2}^{\star} \Delta t \\
\left\langle r_{p} x_{3}-\gamma_{n}\right\rangle_{\varepsilon}-r_{p} x_{3}=0
\end{array}\right\}
$$

$$
\boldsymbol{r}_{\text {slip }}^{\varepsilon}=\left\{\begin{array}{c}
-\gamma_{1} \\
-\gamma_{2} \\
-\gamma_{n} \\
\gamma_{1} \sqrt{\dot{w}_{1}^{2}+\dot{w}_{2}^{2}}+\mu \gamma_{n} \dot{w}_{1} \\
\gamma_{2} \sqrt{\dot{w}_{1}^{2}+\dot{w}_{2}^{2}}+\mu \gamma_{n} \dot{w}_{2} \\
\left\langle r_{p} x_{3}-\gamma_{n}\right\rangle_{\varepsilon}-x_{3}=0
\end{array}\right\}
$$

where $r_{p}$ is a constant to ensure units of force are correct. We use $E L_{c}$ with $E$ being the elasticity modulus and $L_{c}$ the characteristic length of the mesh. Figure 1 shows the frictional cone with conditions.

Of course, this modification has the intended limit:

$$
\lim _{\varepsilon \rightarrow 0}\langle\bullet\rangle_{\varepsilon}=\langle\bullet\rangle
$$

with $\langle\bullet\rangle$ being the unit ramp function. The graph of $\langle\bullet\rangle_{\varepsilon}$ is shown in Fig. 2 and the resulting normal behavior is shown in Fig. 3 .

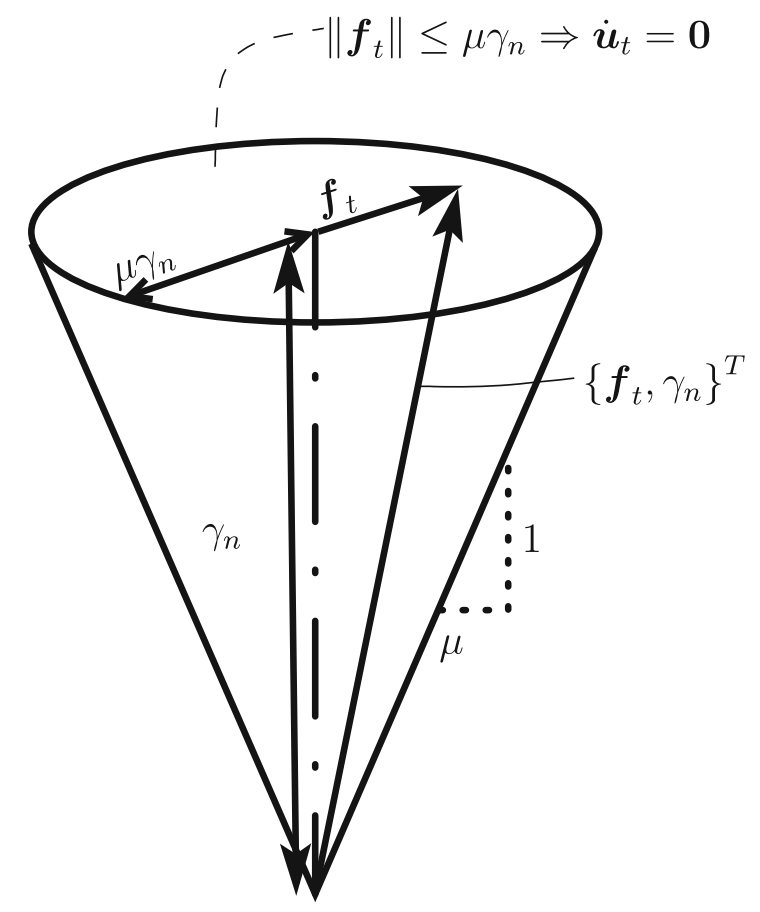

Fig. 1 Frictional cone with relevant quantities

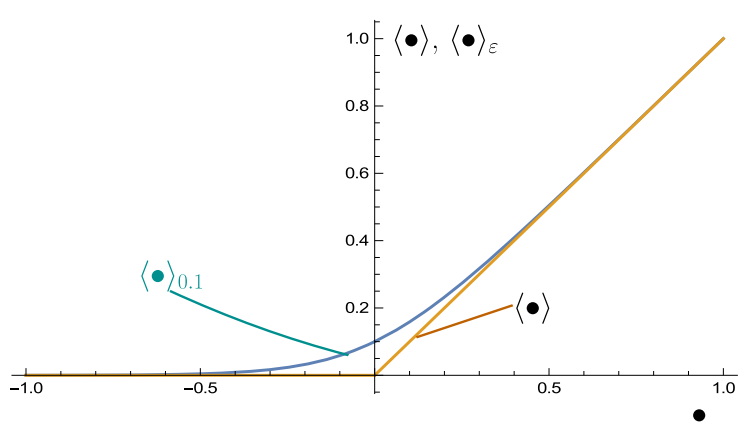

Fig. 2 Graph of $\langle\bullet\rangle_{\varepsilon}$

\section{Generalization for a 3D contact element}

For contact discretization in 3D, we use the node-toface method (e.g. Zavarise and De Lorenzis 2009; Wriggers and Haraldsson 2003). A single pass algorithm is adopted. For detection, we use an incident node averaged normal to chose a main target edge or face, cf. Areias (2003) and Areias et al. (2004). This averaged normal is only used for detection of the first edge or face and not used for contact enforcement, see also Areias et al. (2015). For the contact elements, we 


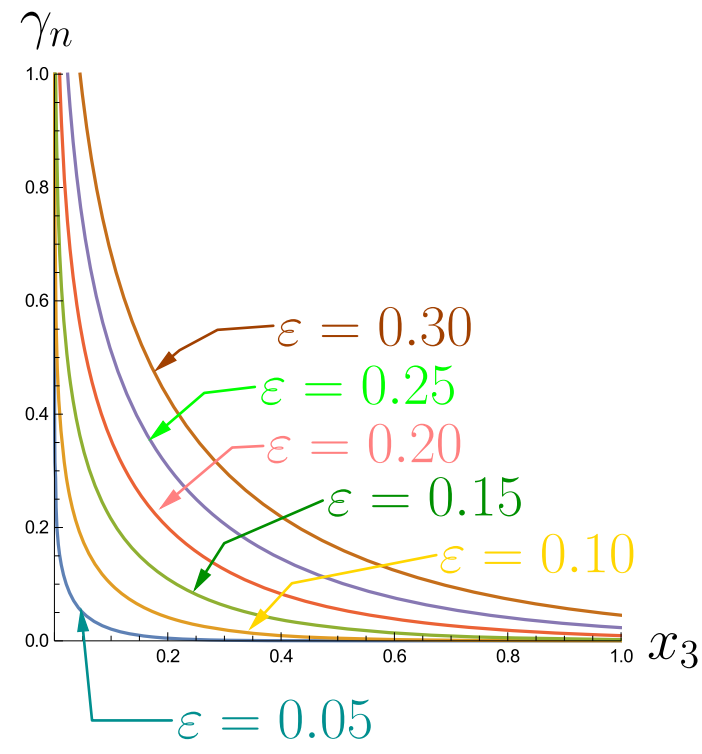

Fig. 3 Normal behavior as a function of $\varepsilon$

allow more than one target edge or face to share an incident node. Further details are provided in Areias et al. (2015). The generalization of the previous formulation for any geometry is performed by use of the force power in local and global frames (see Fig. 4). Implicit determination of kinematic quantities is performed. We use $\boldsymbol{f}_{c l}$ as the contact force in the local frame (as in Sect. 2), $\boldsymbol{f}_{c g}$ as the contact force in the global frame. Conjugate to these are the velocity in the local frame, $\dot{\boldsymbol{u}}$ and in the global frame, $\dot{\boldsymbol{u}}_{g}$. We also

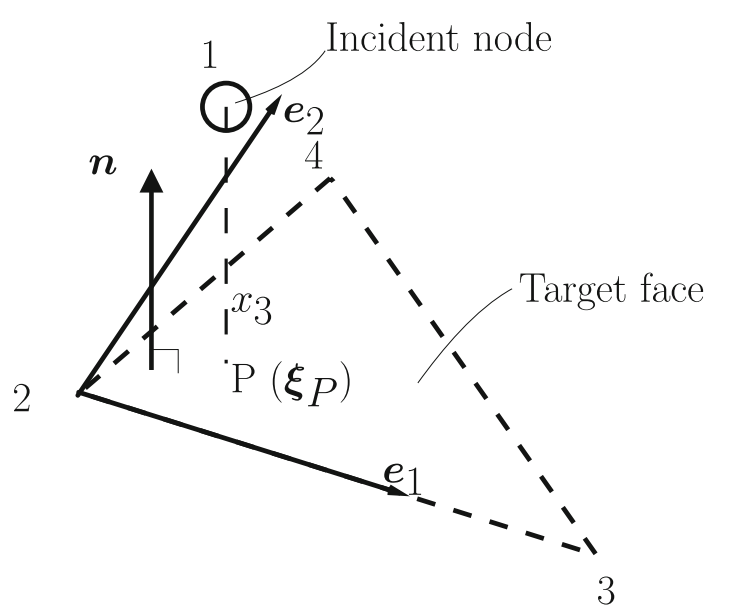

Fig. 4 Tetrahedron contact element. $P\left(\xi_{P}\right)$ is the projection of the incident node on the target face introduce the generalized rates $\dot{\boldsymbol{p}}_{t}$ and $\dot{p}_{n}$ conjugated to $\boldsymbol{R}_{t}$ and $R_{n}$ where

$$
\boldsymbol{R}_{t}=\left\{\begin{array}{c}
\gamma_{1} \sqrt{\dot{w}_{1}^{2}+\dot{w}_{2}^{2}}+\mu \gamma_{n} \dot{w}_{1} \\
\gamma_{2} \sqrt{\dot{w}_{1}^{2}+\dot{w}_{2}^{2}}+\mu \gamma_{n} \dot{w}_{2}
\end{array}\right\}
$$

and

$$
R_{n}=\left\langle r_{p} x_{3}-\gamma_{n}\right\rangle_{\varepsilon}-x_{3}
$$

We then write the following discrete form:

$$
\begin{aligned}
& \dot{W}=\underbrace{\left\{\begin{array}{c}
\boldsymbol{f}_{c l}=\left\{\begin{array}{l}
\boldsymbol{f}_{t} \\
\gamma_{n}
\end{array}\right\} \\
\boldsymbol{R}_{t} \\
R_{n}
\end{array}\right\} \cdot\left\{\begin{array}{c}
\dot{\boldsymbol{u}}=\left\{\begin{array}{c}
\dot{\boldsymbol{u}}_{t} \\
\dot{u}_{3}
\end{array}\right\} \\
\dot{\boldsymbol{p}}_{t} \\
\dot{p}_{n}
\end{array}\right\}}_{\text {localframe }}=\underbrace{\left\{\begin{array}{c}
\boldsymbol{f}_{c g} \\
\boldsymbol{R}_{t} \\
R_{n}
\end{array}\right\} \cdot\left\{\begin{array}{l}
\dot{\boldsymbol{u}}_{g} \\
\dot{\boldsymbol{p}}_{t} \\
\dot{p}_{n}
\end{array}\right\}}_{\text {globalframe }} \\
& =\underbrace{R_{n} \dot{p}_{n}+\boldsymbol{R}_{t} \cdot \dot{\boldsymbol{p}}_{t}+\boldsymbol{f}_{c g} \dot{\boldsymbol{u}}_{g}}_{\text {globalframe,discreteform }}
\end{aligned}
$$

The global velocity $\dot{\boldsymbol{u}}_{g}$ is defined using a global common frame. The two local terms $\dot{\boldsymbol{u}}_{t}$ and $\dot{u}_{3}$ are calculated from $\dot{\boldsymbol{u}}_{g}$ as later described. In $(19,20), \dot{p}_{n}$ and $\dot{\boldsymbol{p}}_{t}$ are velocity-like virtual quantities which are defined in the tetrahedron element as a whole. Introducing a capital $K$ as the local node counter for the contact element shown in Fig. 4, we have an equivalence between the internal product of interpolated quantities and the nodal values of these quantities:

$\boldsymbol{f}_{c g} \cdot \dot{\boldsymbol{u}}_{g}=\sum_{K=1}^{4} \boldsymbol{f}_{c g}^{K} \cdot \dot{\boldsymbol{u}}_{g}^{K}$

Using the classical contact interpolation for $\dot{\boldsymbol{u}}_{g}$, we have (see also Fig. 4):

$\dot{\boldsymbol{u}}_{g}=\dot{\boldsymbol{u}}_{1}-\dot{\boldsymbol{u}}_{P}=\sum_{K=1}^{4} N_{K}\left(\boldsymbol{\xi}_{P}\right) \dot{\boldsymbol{u}}_{K}$

where $N_{K}\left(\xi_{P}\right)$ are the shape functions of nodes evaluated at $\xi_{P}, N_{1}\left(\xi_{P}\right)=1$. Parent-domain coordinates $\xi_{P}$ correspond to the projection of the contact node on the triangular face. Further details are given in Areias et al. (2015). The power equivalence (19) allows the definition of nodal forces $f_{c g}^{K}$ as: 
$\boldsymbol{f}_{c g}^{K}=N_{K}\left(\boldsymbol{\xi}_{P}\right)\left(\frac{\partial \dot{\boldsymbol{u}}}{\partial \dot{\boldsymbol{u}}_{g}}\right)^{T} \boldsymbol{f}_{c l}$

using (19) and the transformation matrix $\frac{\partial \dot{\boldsymbol{u}}}{\partial \dot{\boldsymbol{u}}_{g}}$, which is determined from the explicit relation between $\dot{\boldsymbol{u}}=$ $\left\{\begin{array}{c}\dot{\boldsymbol{u}}_{t} \\ \dot{g}\end{array}\right\}$ and $\dot{\boldsymbol{u}}_{g}$. Using a normal $\boldsymbol{n}$ to the triangular face represented in Fig. 4 it is obvious, from Fig. 4, that

$\dot{u}_{3}=\boldsymbol{n} \cdot \dot{\boldsymbol{u}}_{g}$

$\dot{\boldsymbol{u}}_{t}=\left\{\begin{array}{l}\boldsymbol{e}_{1} \cdot \dot{\boldsymbol{u}}_{g} \\ \boldsymbol{e}_{2} \cdot \dot{\boldsymbol{u}}_{g}\end{array}\right\}$

where $\boldsymbol{e}_{1}$ and $\boldsymbol{e}_{2}$ are defined from the triangle edges. We note that $\dot{\boldsymbol{u}}_{t} \neq[\boldsymbol{I}-\boldsymbol{n} \otimes \boldsymbol{n}] \dot{\boldsymbol{u}}_{g}$ since we require a 2D tangential velocity vector here. We then have

$$
\left(\frac{\partial \dot{\boldsymbol{u}}}{\partial \dot{\boldsymbol{u}}_{g}}\right)^{T}=\left[\begin{array}{lll}
\boldsymbol{e}_{1} \mid & \boldsymbol{e}_{2} \mid & \boldsymbol{n}
\end{array}\right]
$$

For each node in contact (incident node, as depicted in Fig. 4) the target face is identified, and the elements formed. Then the global force (23) is determined and its Jacobian calculated. Forces and the corresponding Jacobian are calculated for this geometry by
Mathematica (Wolfram Research Inc. 2007) with the Acegen add-on Korelc (2002). Source code (Mathematica and Fortran 2003) is available in Github Areias (2018).

\section{Examples}

Examples are performed in SimPlas (Areias, SimPlas) with the current 3D element being open-source (Areias 2018).

\subsection{Raous rectangle}

This 2D problem due to Raous et al. (1988) and solved by Kanno et al. (2006), is here reproduced with a 2D variant of the proposed algorithm. It consists of an elastic solid ( $E=130 \mathrm{GPa}$ and $v=0.2)$ in plane strain. The solid is discretized in 512 quadrilateral $\bar{B}$ (cf. Simo et al. 1985) finite elements. Figure 5 presents the relevant data. In this figure, the deformed mesh, as well as the contact forces at the contact surface are shown. In Kanno et al. (2006) a sophisticated SOCLCP problem formulation is proposed and then solved by a smooth solver. Compared with the present algorithm, there is a close agreement between the results, as Fig. 6 shows. This Figure presents the
Fig. 5 Raous rectangle: relevant data and magnified deformation with the contact forces contour plot

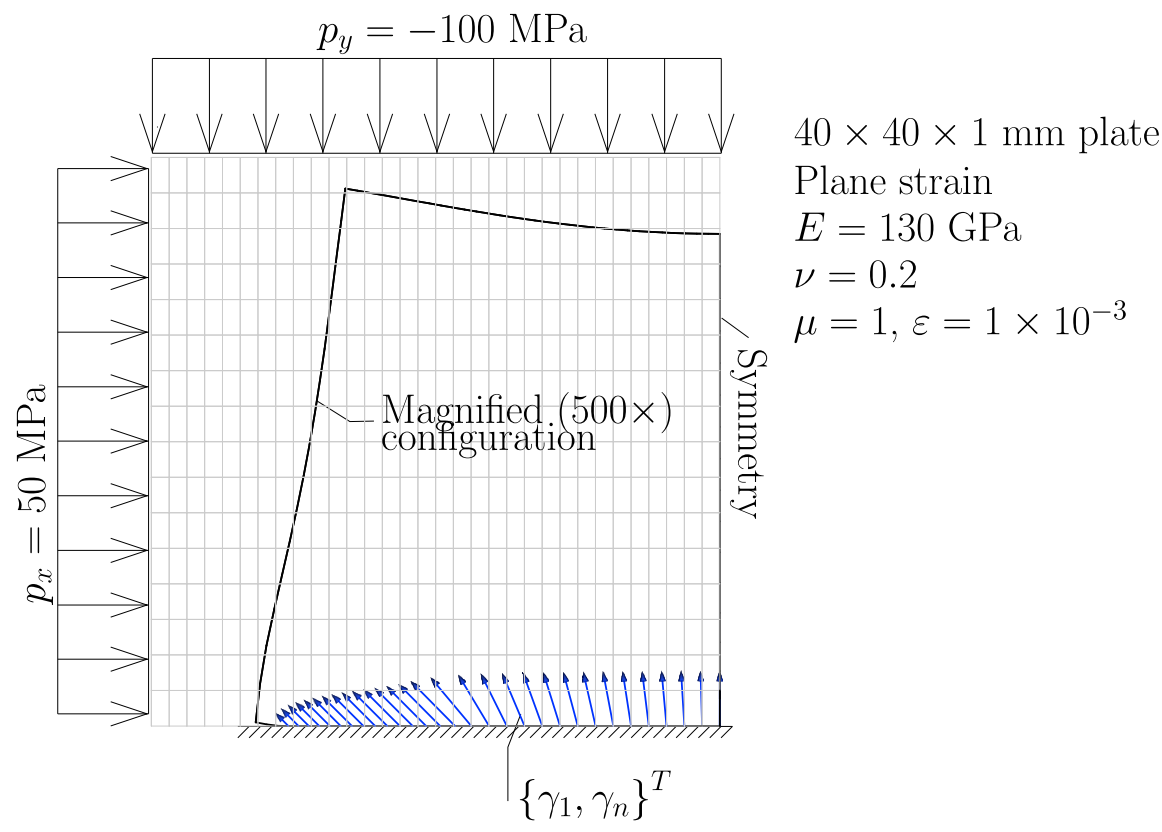


Fig. 6 Raous rectangle: comparison with the formulation using conecomplementarity by Kanno et al. (2006)

Fig. 7 Raous rectangle: convergence of tangential stress with $\xi$
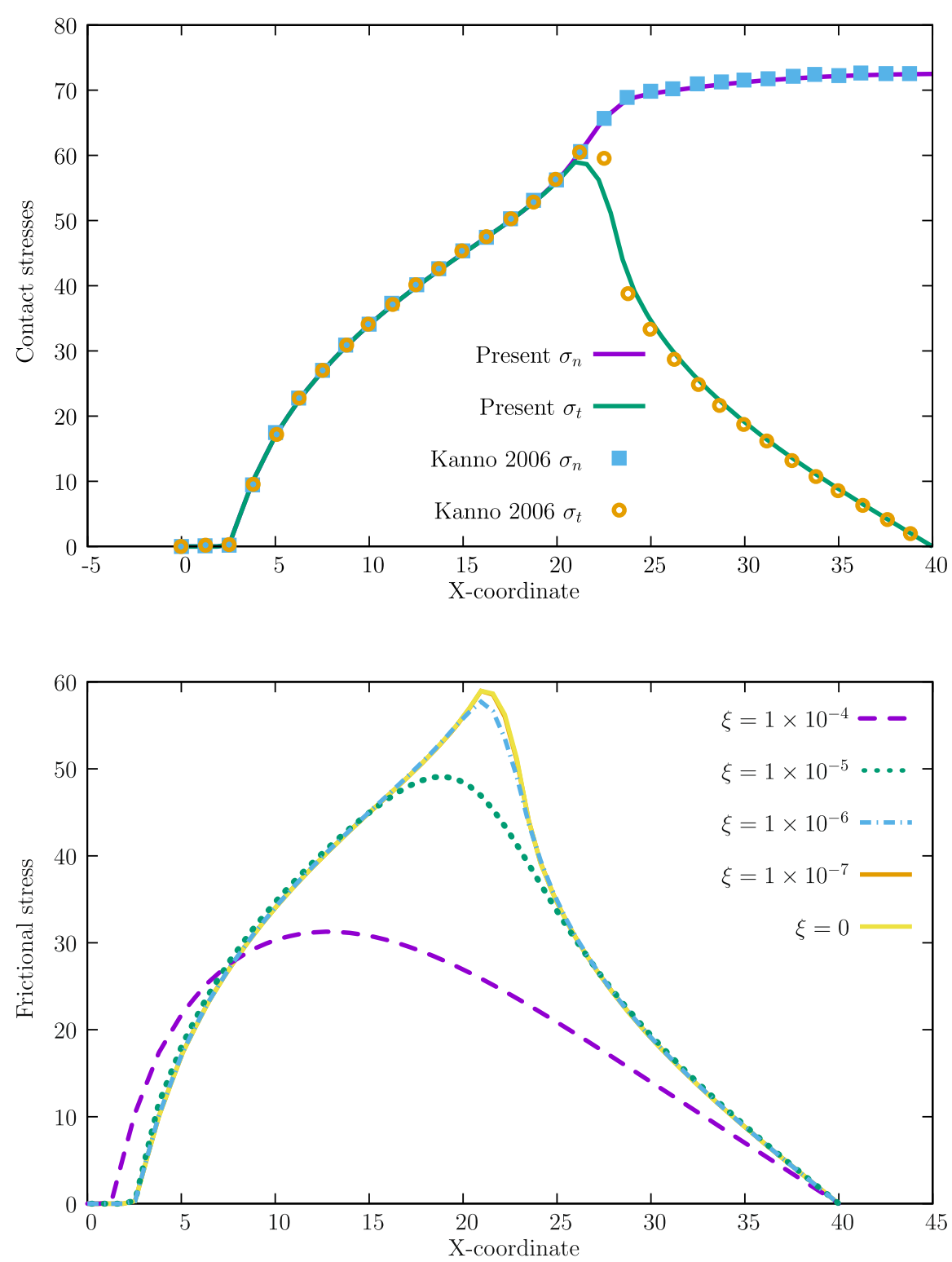

normal and shear stresses ( $\sigma_{n}$ and $\sigma_{t}$, respectively) as functions of the $\mathrm{x}$-coordinate of the contact segment. In terms of convergence of the convex-combination parameter $\xi$, Fig. 7 presents the results. We note that below $\xi=1 \times 10^{-7}$ a correct solution is obtained and a sharp shear stress distribution corresponding to the original Coulomb frictional problem $(\xi=0)$ is achieved. When compared with (Kanno et al. 2006), the present algorithm is very straightforward and easy to implement.

\subsection{Sweeping test}

A sweeping test is proposed here to include the significant ingredients in terms of state transition:

1. Normal direction transition

2. Transition from stick to slip

3. Transition from slip to stick

4. Inversion of sense in slipping 


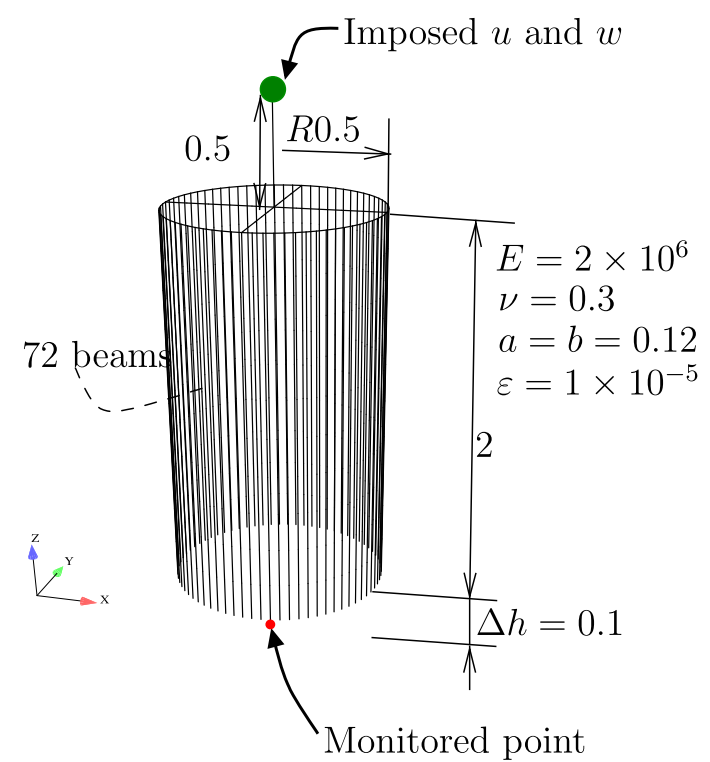

\section{$\underline{\text { Consistent Units }}$}

Imposed $u$ and $w$ :

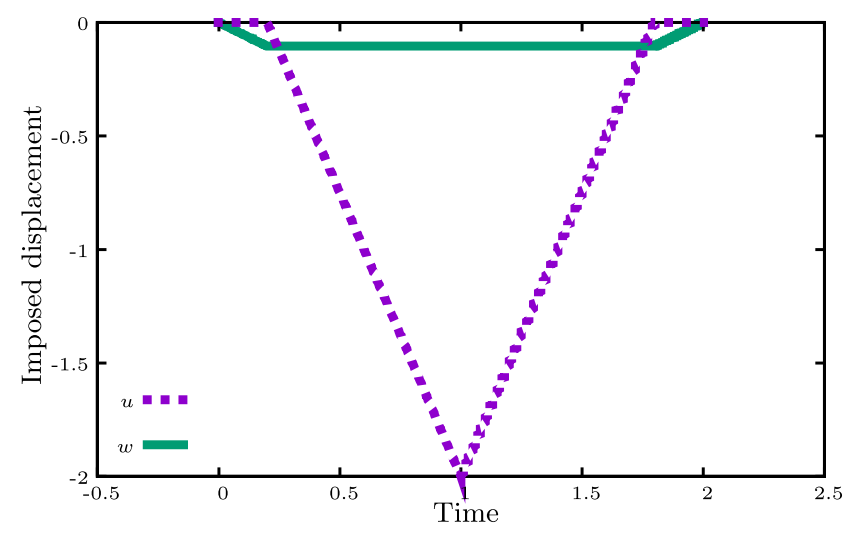

(a) Sweeping test: relevant data.

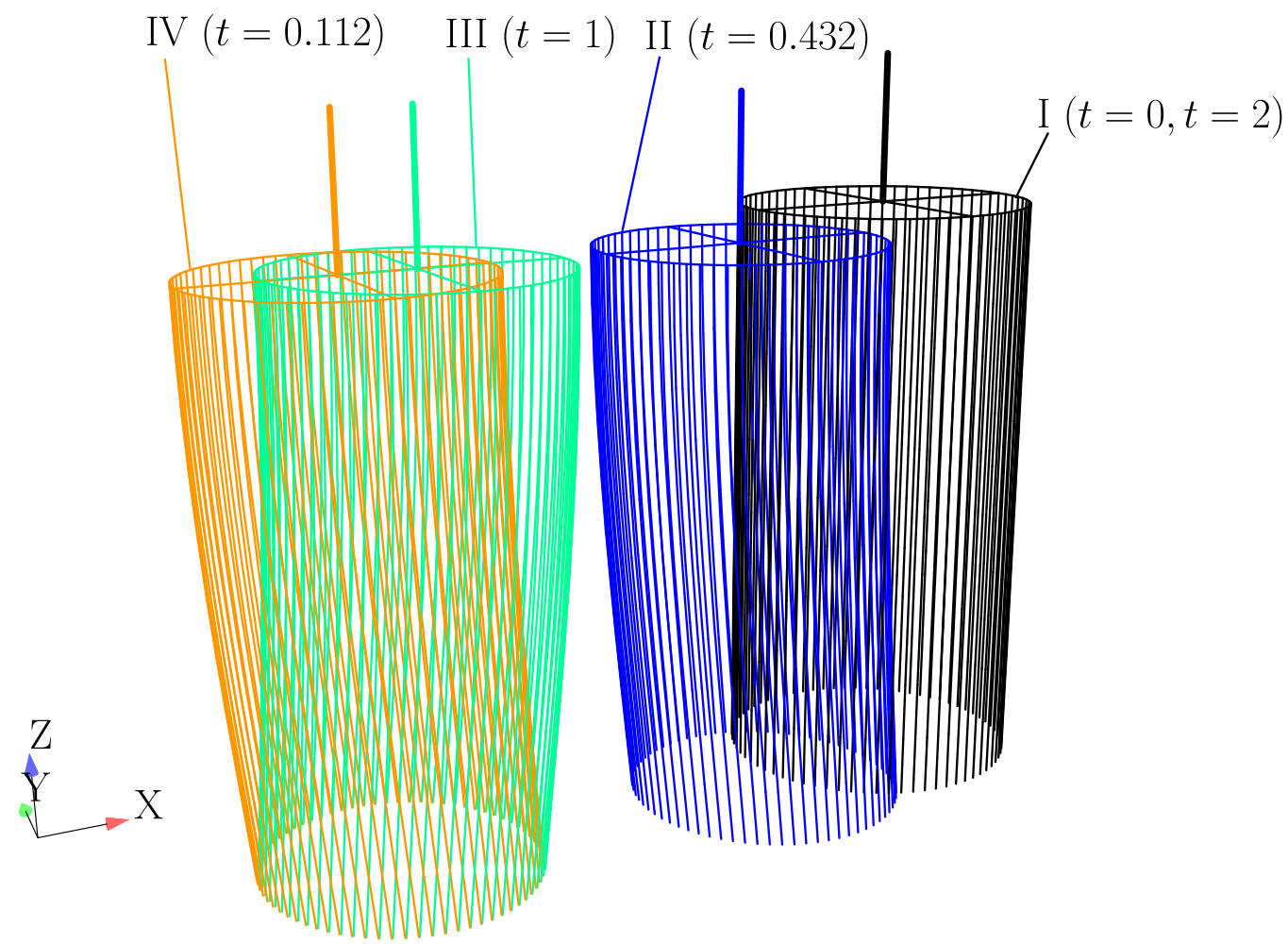

(b) Sweeping test: sequence of configurations.

Fig. 8 Sweeping test: relevant geometrical data and properties and sequence of configurations 


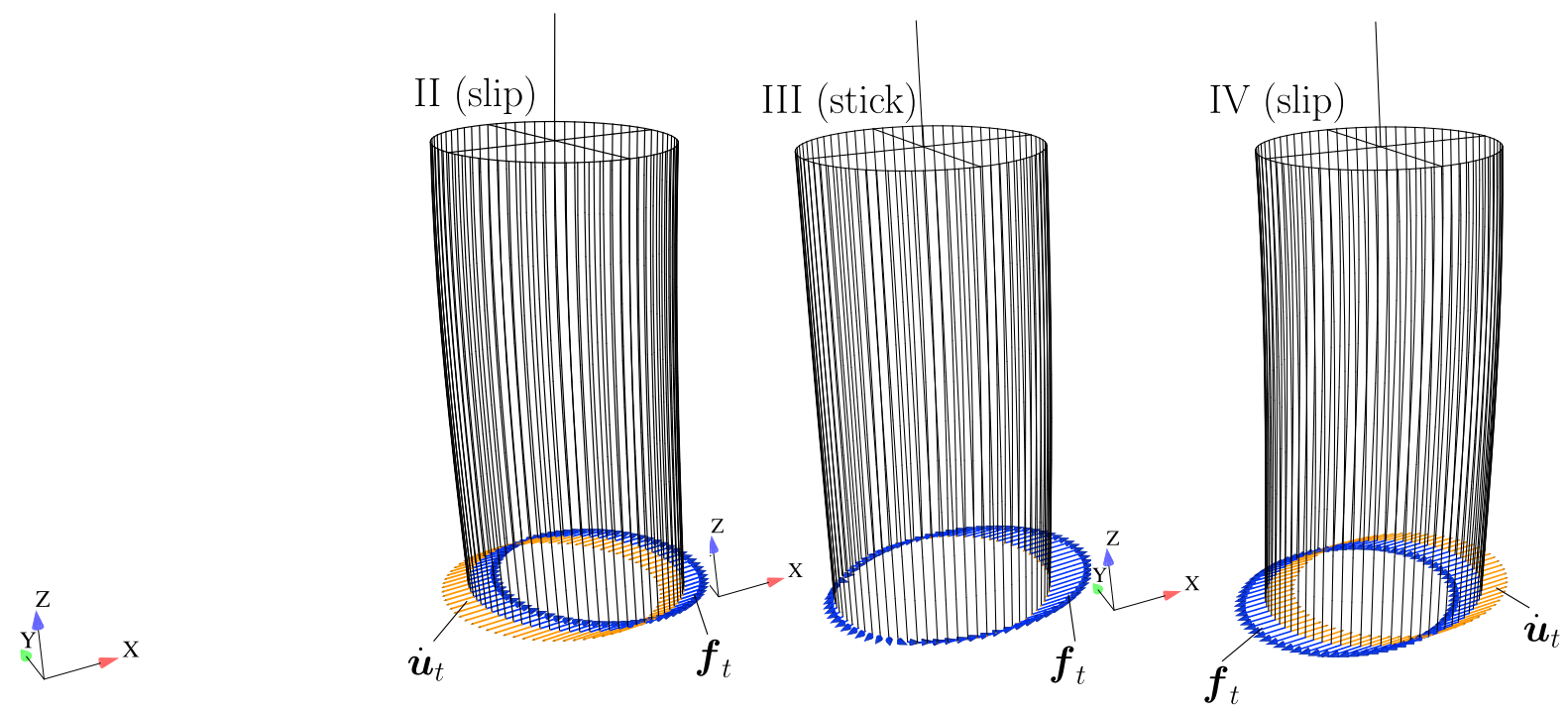

Fig. 9 Sweeping test: configurations with slip and stick

Fig. 10 Sweeping test: displacements of monitored node (cf. Fig. 8a)

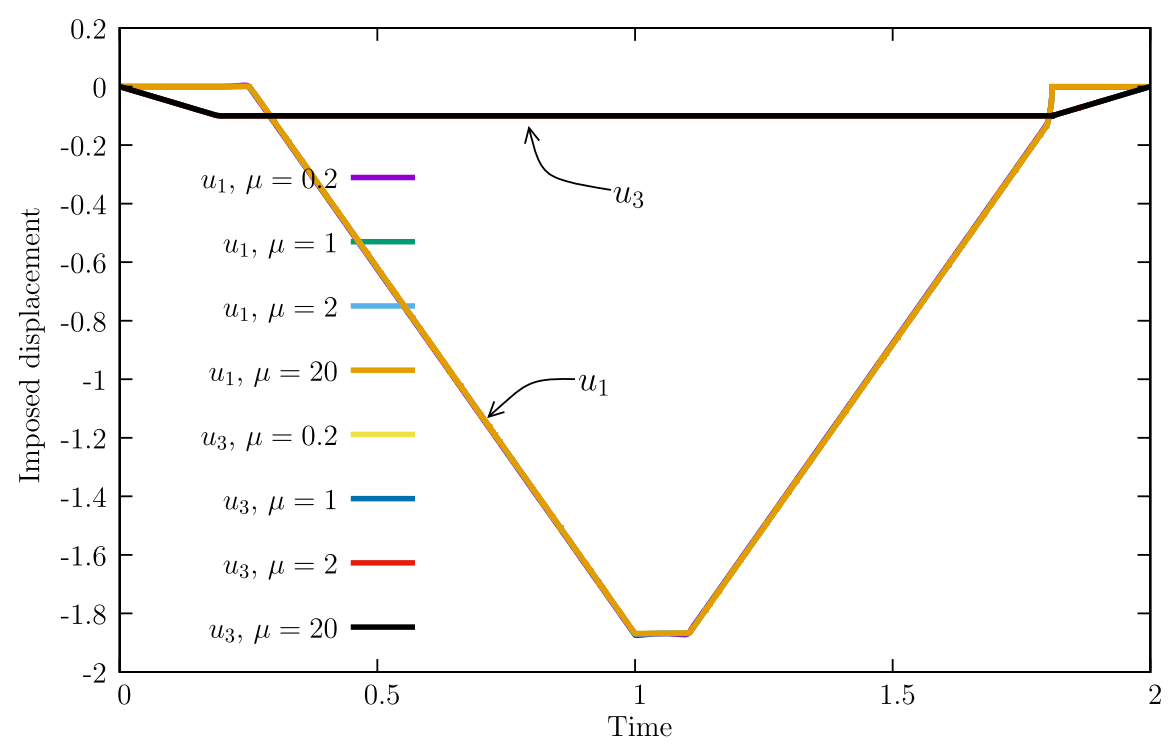

Relevant data for this problem is shown in Fig. 8a and a sequence of four configurations is shown in Fig. 8b. We test four values of the friction coefficient $\mu: \mu=0.2,1,2,20$. Since the normal force is highly dependent on the friction coefficient, displacement results are relatively independent on the friction coefficient. We identify configurations where all points are slipping (II and IV) in sticking and others where all points are sticking (II), cf. Figure 9. This is confirmed by the displacements of the monitored node, see Fig. 10. Between $t=1$ and $t=1.107$ (consistent units), sticking occurs for all values of $\mu$. Observing the reactions in Fig. 11, we can conclude that the higher values of $\mu$ induce a lower normal force, as well as (slightly) higher tangential force. 
Fig. 11 Sweeping test: reactions along directions $x$ (tangential direction) and $z$ (normal direction)

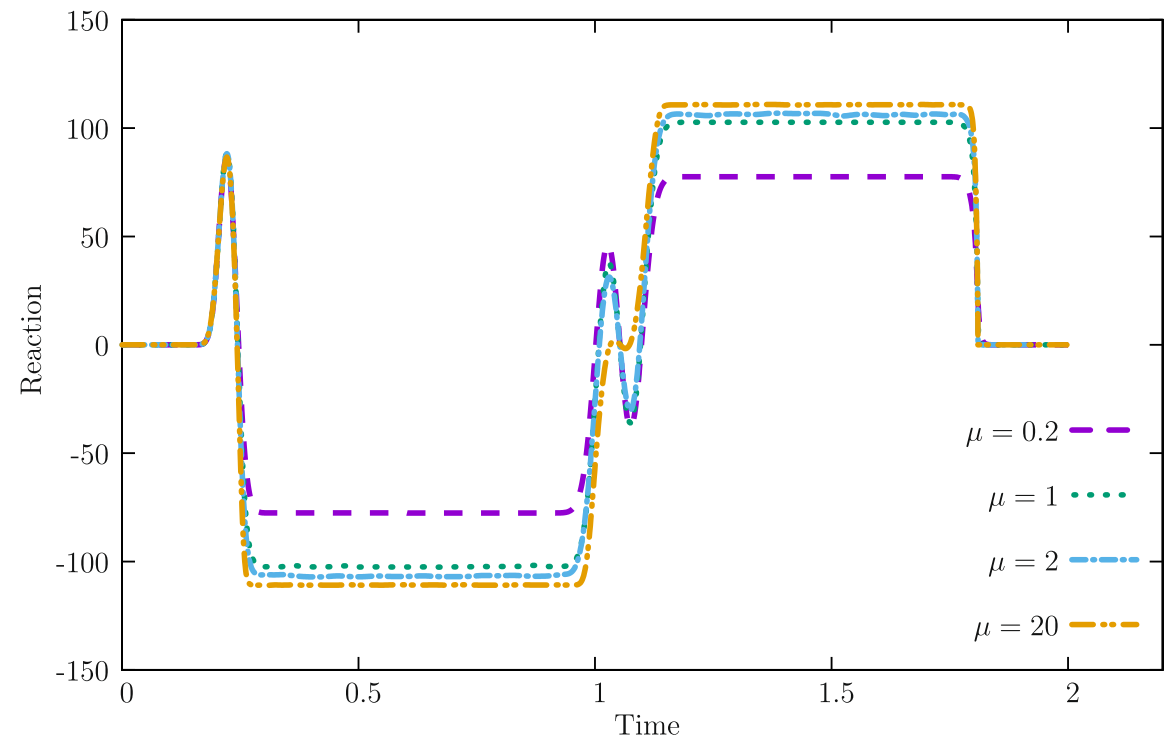

(a) Tangential reactions (imposed node).

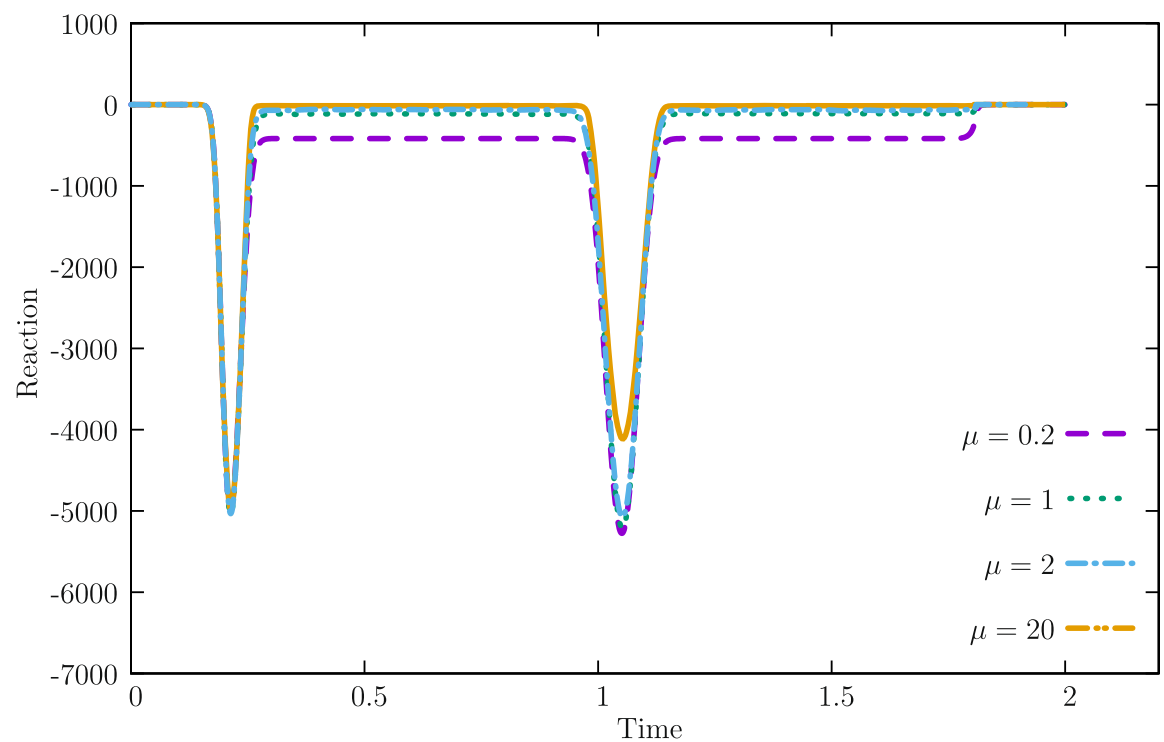

(b) Normal reactions (imposed node).

\subsection{Compressed concentric spheres}

Puso and Laursen (2004a) presented a problem with two concentric spheres and reported failure of the node-to-face algorithm (even in the frictionless case). In this problem, meshes are intentionally mismatched to introduce discontinuities in the gap during sliding motion. Figure 12 presents the relevant data for this problem (we represent the two obstacles that are not visible in the original paper). We solve this problem to a higher value of the imposed displacement. Figure 13 shows the reaction results, compared with the values presented by Puso and Laursen (2004a). We note that for the highest value of friction coefficient $(\mu=2.0)$ some oscillations appear. The frictionless case also exhibits some oscillations due to unrestricted face sliding. 
Consistent Units

$$
\begin{aligned}
& E=1 \quad \varepsilon=1 \times 10^{-4} \\
& \nu=0.3 \quad \text { Neo-Hookean constitutive law } \\
& \Delta t=3 \times 10^{-4}
\end{aligned}
$$
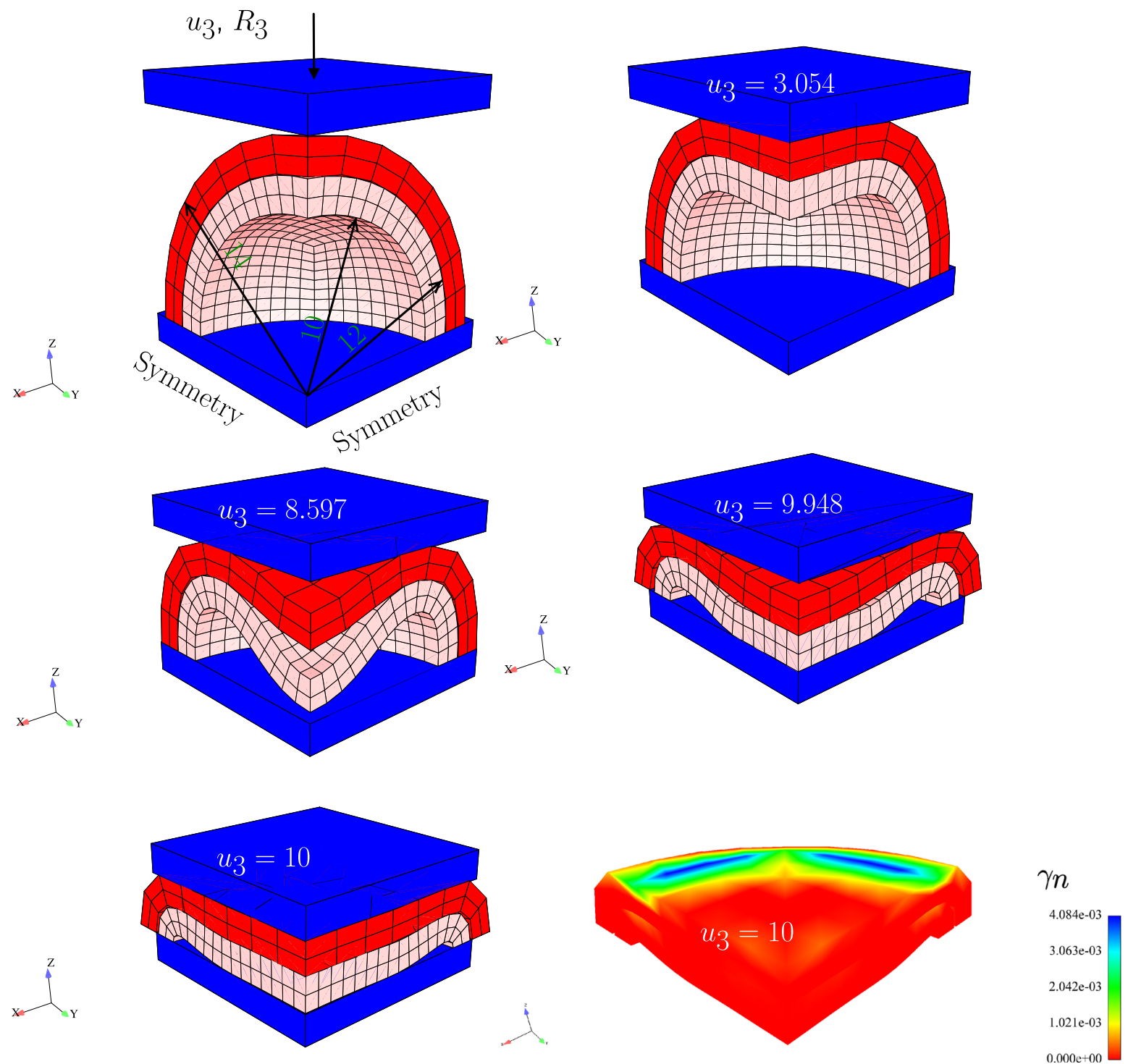

Fig. 12 Compressed concentric spheres: relevant data and sequence of configurations 
Fig. 13 Compressed concentric spheres: displacement/reaction results, compared with (Puso and Laursen 2004a). We test $\mu=0.0, \mu=0.5$ and $\mu=2.0$

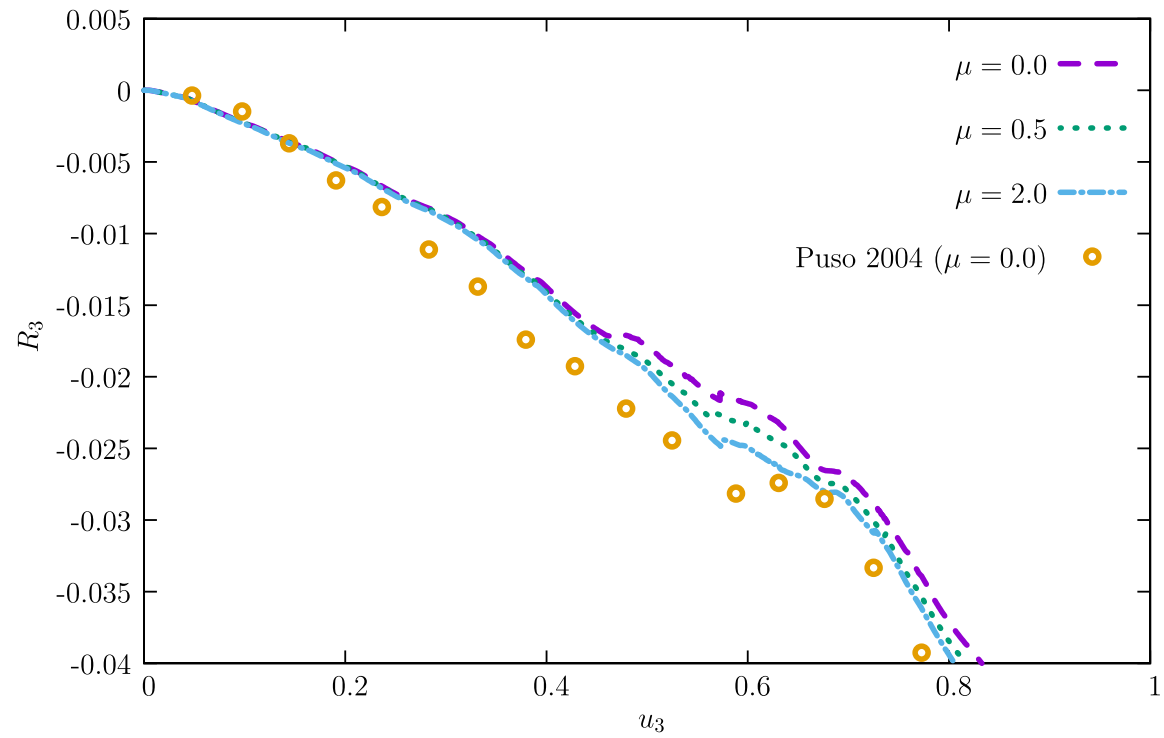

\section{Conclusions}

We introduced a two-stage contact algorithm (stick and slip stages) based on a mixed formulation where three additional degrees-of-freedom corresponding to the contact forces are considered. Since the normal force (Kanno et al. 2006) is non-smooth, we regularize it by means of replacement function depending on one scalar parameter. This formulation and technique were shown to be successful for demanding problems, with high coefficients of friction being used, while retaining the sharpness of the original problem. Three problems were solved, including the concentric spheres including friction, which shows the general applicability of the approach.

Acknowledgements This work was supported by FCT, through IDMEC, under LAETA, project UID/EMS/50022/ 2019.

Data Availability The data required to reproduce these findings is available to download from https://github.com/ PedroAreias/Contact3DElement/.

\section{References}

Alart, P., Curnier, A.: A mixed formulation for frictional contact problems prone to Newton like methods. Comput. Methods Appl. Mech. 92, 353-375 (1992)
Amontons, G.: Sur les frottements des machines. Académie Royale des Sciences, Paris, France, Troiséme (1732) edition, pp. 104-109 (1699)

Anitescu, C., Atroshchenko, E., Alajlan, N., Rabczuk, T.: Artificial neural network methods for the solution of second order boundary value problems. CMC: Comput. Mater. Continua 59(1), 345-359 (2019)

Areias, P.: Simplas. http://www.simplassoftware.com. Portuguese Software Association (ASSOFT) registry number 2281/D/17

Areias, P.: Finite element technology, damage modeling, contact constraints and fracture analysis. Doutoramento, FEUP - Faculdade de Engenharia da Universidade do Porto, Rua Dr. Roberto Frias s/n 4200-465 Porto, Portugal (2003). www.fe.up.pt

Areias, P.: 3D contact source code. https://github.com/ PedroAreias/Contact3DElement/tree/master (2018)

Areias, P., César de Sá, J.M.A., Conceição António, C.A.: Algorithms for the analysis of 3D finite strain contact problems. Int. J. Numer. Methods Eng. 61, 1107-1151 (2004)

Areias, P., Pinto da Costa, A., Rabczuk, T., Queirós de Melo, F.J.M., Dias-da Costa, D., Bezzeghoud, M.: An alternative formulation for quasi-static frictional and cohesive contact problems. Comput. Mech. 53, 807-824 (2014)

Areias, P., Rabczuk, T., Queirós de Melo, F.J.M., César de Sá, J.: Coulomb frictional contact by explicit projection in the cone for finite displacement quasi-static problems. Comput. Mech. 55(1), 57-72 (2015)

Chen, C., Mangasarian, O.L.: Smoothing methods for convex inequalities and linear complementarity problems. Math. Program. 71(1), 51-69 (1995)

Chen, C., Mangasarian, O.L.: A class of smoothing functions for nonlinear and mixed complementarity problems. Comput. Optim. Appl. 5, 97-138 (1996) 
Coulomb, C.-A.: Théorie des machines simples, en ayant égard au frottement de leurs parties et à roideur des cordages. Imprimerie de Huzard-Courcier, Rue du Jardinet, No 12, Paris, France, Nouvelle Édition edition (1821)

De Saxcé, G., Feng, Z.-Q.: The bipotential method: a constructive approach to design the complete contact law with friction and improved numerical algorithms. Math. Comput. Model. 28(4-8), 225-245 (1998)

Kanno, Y., Martins, J.A.C., Pinto da Costa, A.: Three-dimensional quasi-static frictional contact by using second-order cone linear complementarity problem. Int. J. Numer. Methods Eng. 65, 62-83 (2006)

Khenous, H.B., Laborde, P., Renard, Y.: Mass redistribution method for finite element contact problems in elastodynamics. Eur. J. Mech. A/Solids 27, 918-932 (2008)

Kikuchi, N., Oden, J.T.: Contact Problems in Elasticity. A Study of Variational Inequalities and Finite Element Methods. volume 8 of SIAM Studies in Applied and Numerical Mathematics. SIAM Studies in Applied Mathematics, Philadelphia (1988)

Korelc, J.: Multi-language and multi-environment generation of nonlinear finite element codes. Eng. Comput. 18(4), 312-327 (2002)

Laursen, T.A.: Computational Contact and Impact Mechanics. Fundamentals of Modeling Interfacial Phenomena in Nonlinear Finite Element Analysis. Springer, Berlin (2002)

Martins, J.A.C., Simões, F.M.F., Gastaldi, F., Monteiro Marques, M.D.P.: Dissipative graph solutions for a 2 degree-offreedom quasistatic frictional contact problem. Int. J. Eng. Sci. 33(13), 1959-1986 (1995)

Pennestri, E., Rossi, V., Salvini, P., Valentini, P.P.: Review and comparison of dry friction force models. Nonlinear Dyn. 83, 1785-1801 (2016)

Puso, M.A., Laursen, T.A.: A mortar segment-to-segment contact method for large deformation solid mechanics. Comput. Methods Appl. Mech. 193, 601-629 (2004a)
Puso, M.A., Laursen, T.A.: A mortar segment-to-segment frictional contact method for large deformations. Comput. Methods Appl. Mech. 193, 4891-4913 (2004b)

Raous, M., Chabrand, P., Lebon, F.: Numerical methods for frictional contact problems and applications. J. Theor. Appl. Mech. 7(Suppl. 1), 111-128 (1988)

Refaat, M.H., Meguid, S.A.: On the modeling of frictional contact problems using variational inequalities. Finite Elem. Anal. Des. 19(1-2), 29-101 (1995)

Simo, J., Laursen, T.: An augmented Lagrangian treatment of contact problems involving friction. Comput. Struct. 42, 97-116 (1992)

Simo, J.C., Taylor, R.L., Pister, K.S.: Variational and projection methods for the volume constraint in finite deformation elasto-plasticity. Comput. Methods Appl. Mech. 51, 177-208 (1985)

Stewart, D.E.: Finite-dimensional contact mechanics. Philos. Trans. R. Soc. A 359, 2467-2482 (2001)

Wolfram Research Inc. Mathematica. Champaign, IL (2007)

Wriggers, P.: Computational Contact Mechanics. Wiley, New York (2002)

Wriggers, P., Haraldsson, A.: A simple formulation for twodimensional contact problems using a moving friction cone. Commun. Numer. Methods Eng. 19, 285-295 (2003)

Zavarise, G., De Lorenzis, L.: The node-to-segment algorithm for 2D frictionless contact: classical formulation and special cases. Comput. Methods Appl. Mech. 198, 3428-3451 (2009)

Publisher's Note Springer Nature remains neutral with regard to jurisdictional claims in published maps and institutional affiliations. 